\title{
HYPERSONIC LAMINAR/TURBULENT TRANSITION: COMPUTATIONS AND EXPERIMENTS
}

\author{
E. Orlik ${ }^{1,2}$, V. Kornilov ${ }^{2}$, M. Ferrier ${ }^{1}$, I. Fedioun ${ }^{1}$, \\ and D. Davidenko ${ }^{1}$ \\ ${ }^{1} \mathrm{ICARE}-\mathrm{CNRS}$ \\ 1C Av. de la Recherche Scientifique, 45071 Orléans cedex 2, France \\ ${ }^{2}$ ITAM \\ Russian Academy of Sciences \\ Institutskaya Str. 4/1, Novosibirsk 630090, Russia
}

\begin{abstract}
In order to predict the laminar/turbulent transition on a hypersonic vehicle forebody at Mach numbers 4 and 6, the three-dimensional (3D) modal linear stability analysis is applied, coupled with the $e^{N}$ method. Nevertheless, $N$ factors are unknown for wind tunnel conditions. Experimental investigations have been carried out on a flat plate in the blowdown wind tunnel T-313 of ITAM RAS (Novosibirsk). At $\mathrm{M}_{\infty}=2$ to 6 , the position of laminar/turbulent transition was measured by both Pitot tube and thermocouples. Then, stability analysis allows computing $N$ factors at transition on the flat plate: they are about $3 \sim 4$, typical of conventional wind tunnels. These flat plate correlations can then be used to predict the transition on the forebody in the same wind tunnel. Experiments for the forebody are currently in progress and will allow checking the predicted transition location.
\end{abstract}

\section{INTRODUCTION}

The prediction of laminar/turbulent transition in hypersonic flow is still a challenging task after half a century of intensive research, both experimental and computational. The recent regain of interest for hypersonic air-breathing scramjet-powered vehicles has led to consider the laminar/turbulent transition prediction from an engineering point of view, in particular, on full scale 3D realistic configurations. At the moment, the only method of industrial application, taking into account theoretically (although incompletely) the destabilizing mechanisms, is the modal linear stability theory (LST). Local or nonlocal (i. e.,

This is an Open Access article distributed under the terms of the Creative Commons Attribution-Noncommercial License 3.0, which permits unrestricted use, distribution, and reproduction in any noncommercial medium, provided the original work is properly cited. 
parabolised stability equations (PSE)) theories may be applied as well [1]. They both rely on the $e^{N}$ method and are of comparable efficiency in predicting the position of transition: their difference for a given $N$ may be of the same order of magnitude as the uncertainty on the value of $N$ itself at the transition position. The real interest of nonlocal theories is to open the way for nonlinear theories that allow a deeper understanding of the transition processes. Magnitude $N \approx 10$ is a currently accepted value for the transition to occur in flight conditions. In wind tunnels, this value may be decreased dramatically, and is $a$ priori unknown.

The object of this paper is to show how $N$ factors can be obtained from flat plate transition measurements in a given wind tunnel and how they can be used for the transition prediction on a $3 \mathrm{D}$ forebody.

Experiments were conducted in the blowdown wind tunnel T-313 of ITAM RAS. Transition data on a flat plate have been obtained by V. Kornilov [2] at various Mach and Reynolds numbers. The method and results, together with the $N$ factors determination are reported in section 2 . Section 3 describes the mean flow and stability analysis for a hypersonic forebody in the T-313 conditions, and the transition prediction is discussed. Section 4 gives conclusions and perspectives for experimental investigations on the forebody in T-313 conditions.

\section{FLAT PLATE EXPERIMENTS AND ASSOCIATED $N$ FACTORS}

\subsection{Experimental Setup and Transition Results}

The blowdown wind tunnel T-313 has a $0.6 \times 0.6 \mathrm{~m}$ test section and a Mach number range 2-7. Experiments were carried out on a flat plate with the leading edge having a small bluntness thickness $b$ equal to $0.05 \mathrm{~mm}$. The plate is equipped with 31 holes for static pressure measurement, and with $59 \mathrm{Cr}-\mathrm{C}$ thermocouples.

Flow parameters are:

$-\mathrm{M}_{\infty}=2$ to 6

$-T_{\text {tot }}=300$ to $400 \mathrm{~K}$;

- $P_{\text {tot }}=1.2$ to 9.4 bar;

$-\operatorname{Re}_{u}=11 \cdot 10^{6}$ and $16 \cdot 10^{6} 1 / \mathrm{m} ;$ and

- zero angle of attack.

The laminar/turbulent transition position is detected using a Pitot tube by the change in total pressure measured in the boundary layer. The Pitot tube is considered to lie on the surface when the electrical contact is established. The tube is then moved downstream over the surface. In the present study, a Pitot 
tube with a 0.2-millimeter flattened tip has been used. The same technique will be used to detect the transition on the forebody. The tube measures the average total pressure over its diameter. A previous study [3] has shown that the size of the tips has some effect on the detection of the beginning of transition, but not the end of transition, as shown in Fig. 1. The end of transition can be also detected by the maximum in temperature distribution. A comparison between Pitot tube and thermocouples measurements shows a good agreement. An example is given in Fig. 2 for $\mathrm{M}_{\infty}=$ 2.5. Main results of experiments are shown in Fig. 3. The transition Reynolds number increases both with the free stream Mach number and with the unit Reynolds number. This behavior is well known and agrees with other measurements on flat plate [4-6].

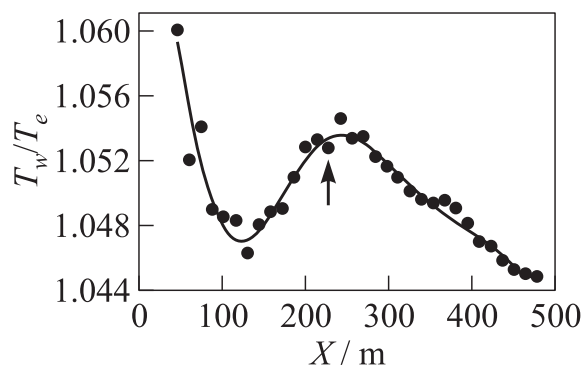

Figure 2 Distribution of normalized wall temperature at $\mathrm{M}_{\infty}=2.5$. The arrow shows the end of transition measured by Pitot tube

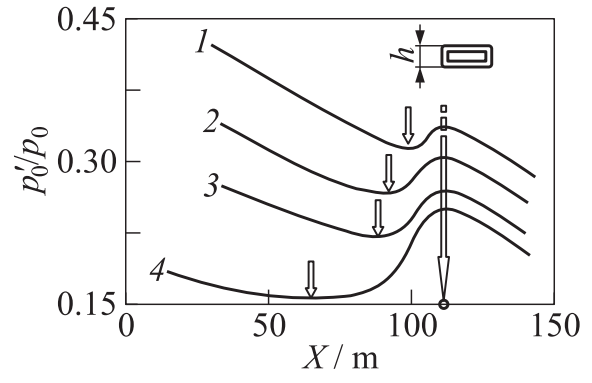

Figure 1 Distribution of normalized Pitot pressure depending on the size of the tube: $1-h=0.62 \mathrm{~mm} ; 2-0.50 ; 3-0.40$; and $4-h=0.17 \mathrm{~mm}\left(\mathrm{M}_{\infty}=2, \operatorname{Re}_{u}\right.$ $\left.=20 \cdot 10^{6} 1 / \mathrm{m}\right)[3]$ 


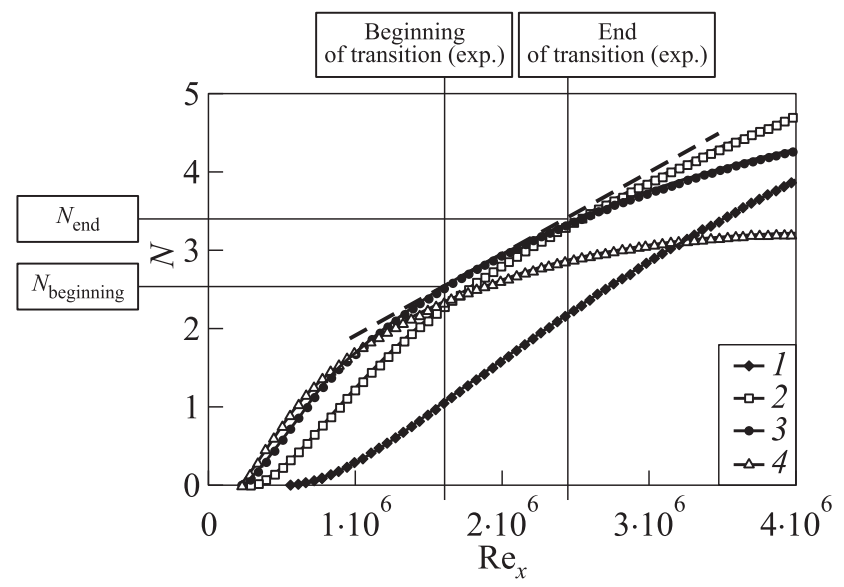

Figure 4 Illustration of determining $N_{\text {beginning }}$ and $N_{\text {end }}$ of transition for different frequencies at $\mathrm{M}_{\infty}=3: 1-10 \mathrm{kHz} ; 2-20 ; 3-30$; and $4-40 \mathrm{kHz}$

Levy-Lees self-similar solution with fully variable thermodynamic and transport properties. The effect of leading edge bluntness is neglected. The profiles equidistant with a 5-millimeter step are discretized with 500 points normal to the wall. The first profile is at a distance of $5 \mathrm{~mm}$ from the leading edge.

The LST is applied to the mean flow for several frequencies, in a fully compressible formalism, using a 3D stability code developed at ICARE [7]. For each frequency $f$, the envelope method is applied to select the direction $\psi=\psi_{\mathrm{M}}$ of propagation of the most unstable $3 \mathrm{D}$ disturbances. The amplification rates $\alpha_{i}$

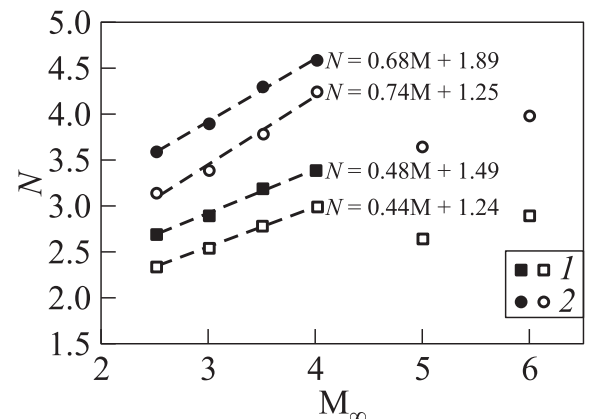

Figure 5 The $N$ factor for beginning (1) and end (2) of transition on a flat plate, T313: closed symbols $-\operatorname{Re}_{u}=16 \cdot 10^{6}$ and open symbols $-\operatorname{Re}_{u}=11 \cdot 10^{6}$ are then integrated to obtain the local total amplification and, hence, the $N$ factor:

$$
N(x, f)=\int_{x_{0}}^{x}-\left.\alpha_{i}(x)\right|_{\psi=\psi_{\mathrm{M}}} d x .
$$

The procedure to obtain $N$ factors for the beginning and end of transition is illustrated in Fig. 4 for the case $\mathrm{M}_{\infty}=3$ and $\operatorname{Re}_{u}=11$. $10^{6} 1 / \mathrm{m}$ : having drawn the $N(x, f)$ curves for several selected frequencies, a straight line (dashed in Fig. 4) is positioned to envelope the curves. The intersection of this line with the 
experimentally determined position of beginning and end of transition gives the corresponding $N$ factors.

At the beginning and end of experimental transition (see Fig. 3), $N$ factors have been found in the range 2.4-4.6 (Fig. 5), typical of conventional wind tunnels. From $\mathrm{M}_{\infty}=4$ to 5 , the main destabilizing process for the flat plate is changed from oblique 1st mode (TS-like waves) to acoustic straight 2 nd mode in Mack's classification [8]. This explains the nonmonotonic behavior of $N$ factors observed in Fig. 5.

\section{TRANSITION ON THE FOREBODY IN T-313: NUMERICAL PREDICTION}

\subsection{Mean Flow Computations}

The full-scale forebody is about $1.3 \mathrm{~m}$ long and has a blunt-nose of radius $5 \mathrm{~mm}$. Upper and lower faces are plane, with angles $+5^{\circ}$ and $-4^{\circ}$ (Fig. 6). A 1:3 scaled model will be tested in the blowdown wind tunnel T-313. The mean flow computation and the stability analysis are done for the wind tunnel conditions, gathered in Table 1.

The mean flow around the forebody is computed with the

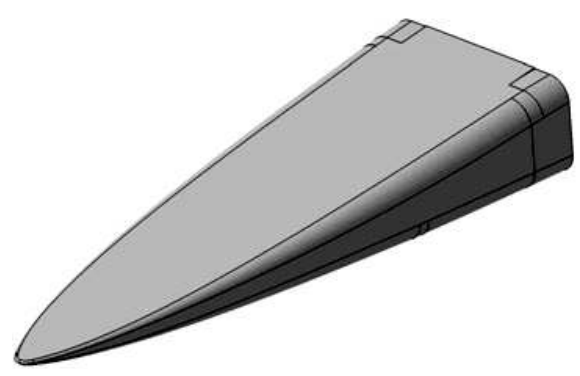

Navier-Stokes commercial code FLUENT ${ }^{\circledR}$. The numerical procedure and flow topology are described in detail in $[9,10]$. An overview of the $2 \cdot 10^{6}$ grid cells computational mesh is shown in Fig. 7. Navier-Stokes computations are used also to prepare experiments for such real 3D geometry. For example, Pitot tube measurements require knowing the boundary layer thickness in order to choose correctly the tips as explained in subsection 1.1. Maps of the boundary layer thickness defined by the total enthalpy criterion (as proposed by Kimmel et al. [11]) are given in Fig. 8 for $\mathrm{M}_{\infty}=4$ and 6 .

Table 1 Flow parameters for the simulation of T-313 test conditions

\begin{tabular}{cccccc}
\hline $\mathrm{M}_{\infty}$ & $P_{\text {st }}, \mathrm{Pa}$ & $T_{\text {st }}, \mathrm{K}$ & $P_{\text {tot }}$, bar & $T_{\text {tot }}, \mathrm{K}$ & $\mathrm{Re}_{u} \cdot 10^{6}, 1 / \mathrm{m}$ \\
\hline 4 & 6617 & 66.9 & 10 & 282 & 48 \\
6 & 511 & 48.7 & 8 & 400 & 9.4 \\
\hline
\end{tabular}




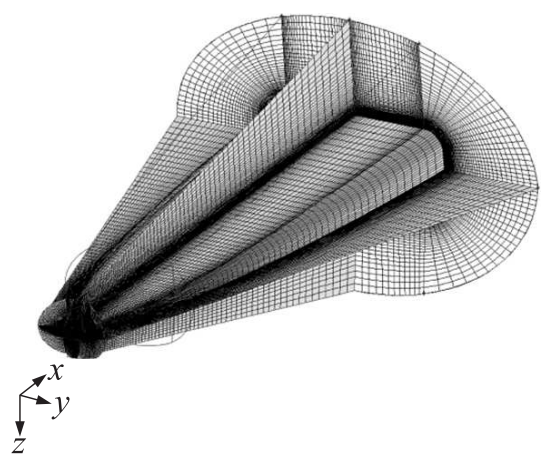

Figure 7 Overview of computational mesh

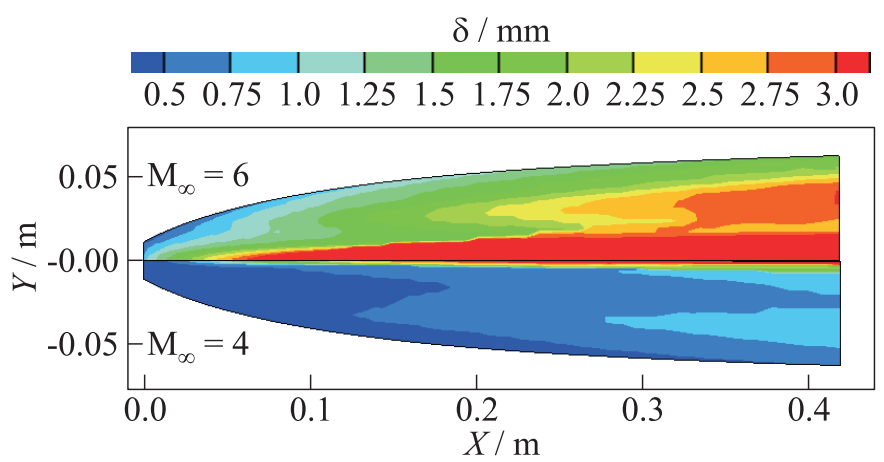

Figure 8 Thickness of boundary layer at $\mathrm{M}_{\infty}=4$ and 6. (Refer Orlik et al., p. 248.)

\subsection{Stability Analysis}

The modal linear stability analysis of the flow on this forebody is performed using the same code as for the flat plate. Anyway, the mean flow is now 3D and the amplification rate has two components $\alpha_{i}$ and $\beta_{i}$ in the streamwise and transversal directions. It is assumed in the calculations that unstable waves are amplified in the direction $\bar{\psi}$ of the group velocity. This gives the closure relation for the dispersion relation in the spatial theory [12]:

$$
\bar{\psi}=\tan ^{-1}\left(\frac{\beta_{i}}{\alpha_{i}}\right) .
$$

The most interesting feature revealed by the stability analysis is the change in the nature of instabilities from Mack's oblique 1 st mode at $\mathrm{M}_{\infty}=4$ to crossflow instability at $\mathrm{M}_{\infty}=6$ (Fig. 9).

This can be observed in Fig. 10 where the angle of propagation of the most unstable waves $\psi_{\mathrm{M}}$ is always less than $80^{\circ}$ at $\mathrm{M}_{\infty}=4$ and reaches higher values 
near the nose at $\mathrm{M}_{\infty}=6$ indicating local crossflow instability. This is related to the topology of the flow which converges towards the symmetry plane and creates counterrotating longitudinal vortices. This has been illustrated and discussed in the 2nd EUCASS conference [13]. Moreover, since the absolute value of the amplification rate $\alpha_{i}$ is higher, the flow is more unstable at $\mathrm{M}_{\infty}=4$ than at $\mathrm{M}_{\infty}=6$.

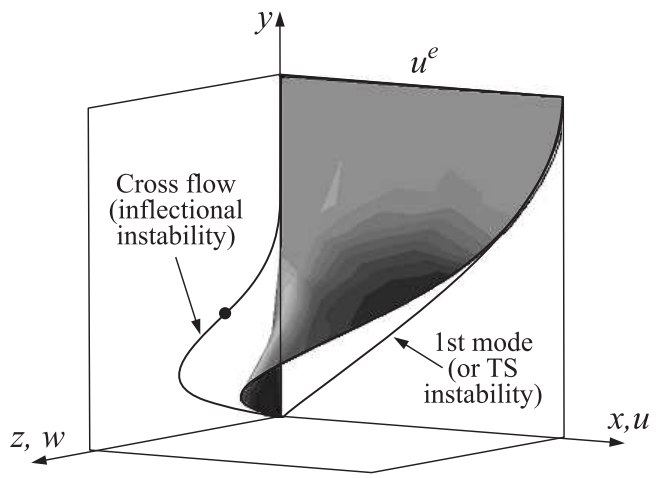

Figure 9 The 3D mean flow: illustration of streamwise (TS) and crossflow instability

\subsection{N Factor Computations on the Three-Dimensional Geometry}

To be coherent with the choice of the local amplification direction Eq. (1), the integration path must be tangent to the group velocity in the computation of $N$ factors:

$$
N(s, f)=\max _{\psi} \int_{s_{0}}^{s} \sqrt{\alpha_{i}^{2}+\beta_{i}^{2}} d \xi .
$$

The numerical procedure is described in detail in $[7,10]$.

Computed $N$ factors for $\mathrm{M}_{\infty}=4$ and 6 are shown in Figs. 11 and 12, respectively. At $\mathrm{M}_{\infty}=4$, the predicted transition based on flat plate data should be close to the nose, as $N$ factors reach quickly values higher than 4 . At $\mathrm{M}_{\infty}=6$, predicted transition should be again close to the nose (Fig. 12a), but in the integration process, both 1st modes (TS-like waves) and crossflow modes which are of different nature are summed up (see Fig. 9). Partial subtracting of the leading edge crossflow instability $\left(\psi_{\mathrm{M}}<85^{\circ}\right.$, Fig. $12 b$, or $\psi_{\mathrm{M}}<80^{\circ}$, Fig. $\left.12 c\right)$ moves significantly the transition position downstream.

\section{CONCLUDING REMARKS AND PERSPECTIVES}

Experimental transition data on a flat plate in the T-313 wind tunnel have been obtained for the Mach number range 2 to 6 . The stability analysis indicates that $N$ factors at transition are about 2.4-4.6.

Regarding future tests on a forebody model in T-313, the following conclusions and previsions can be drawn: at $\mathrm{M}_{\infty}=4$, the transition position is expected 


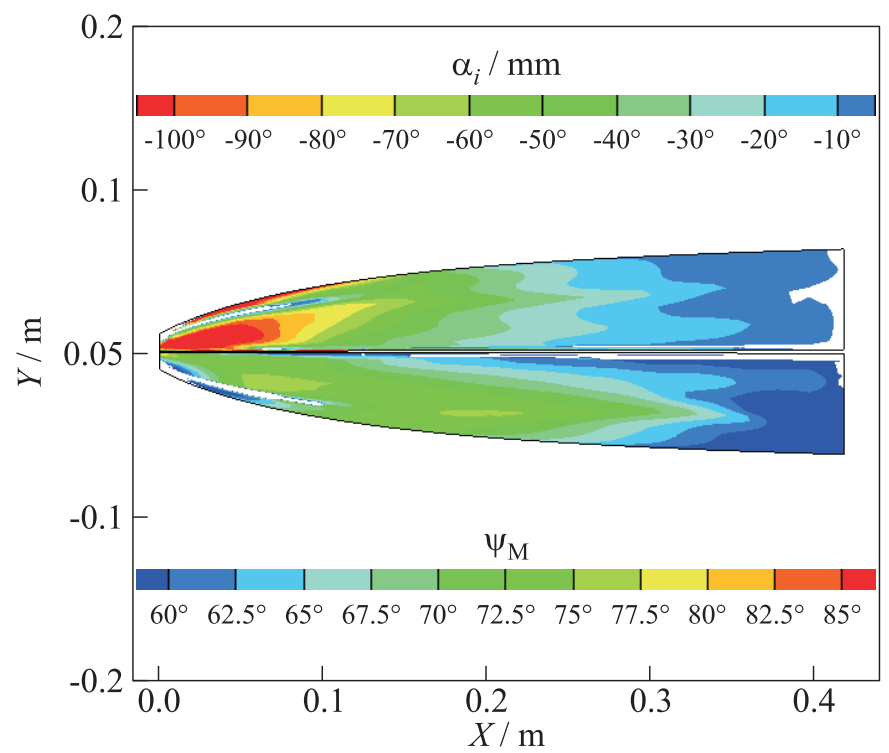

(a)

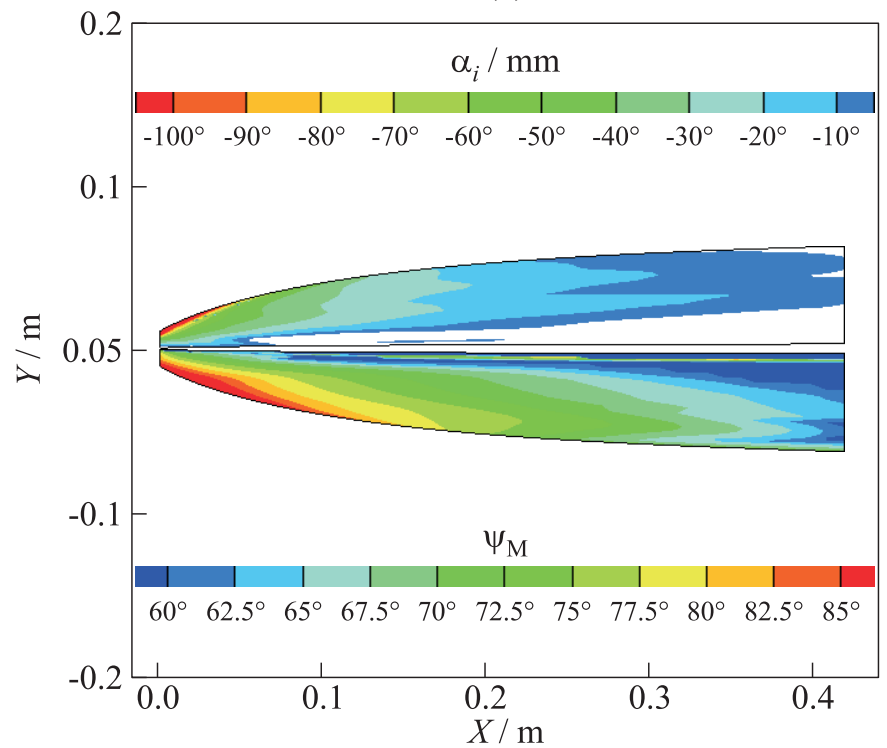

(b)

Figure 10 Computed amplification rates and direction of the most amplified waves at $\mathrm{M}_{\infty}=4(a)$ and $\mathrm{M}_{\infty}=6(b)$. (Refer Orlik et al., p. 250.) 


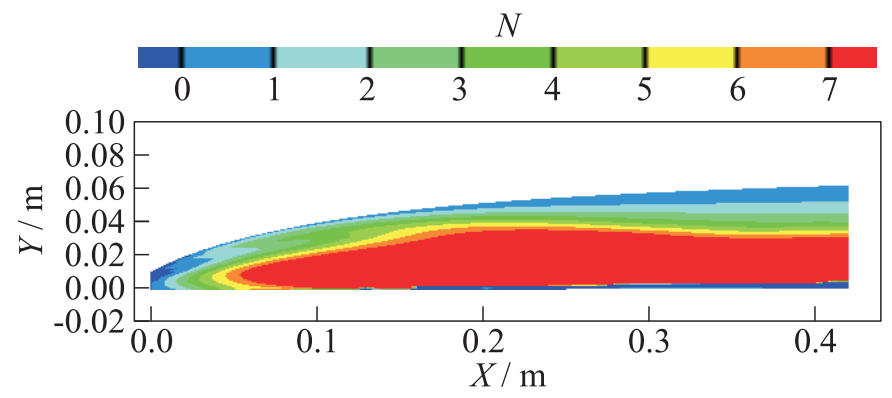

Figure $11 N$ factor of transition at $\mathrm{M}_{\infty}=4$. (Refer Orlik et al., p. 251.)

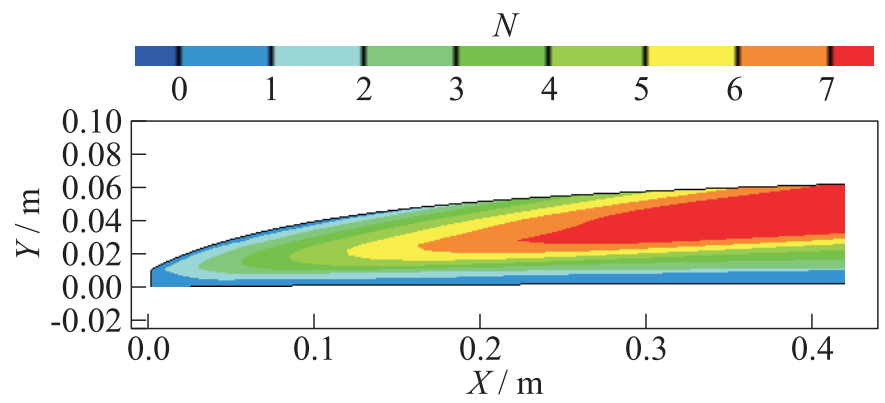

(a)

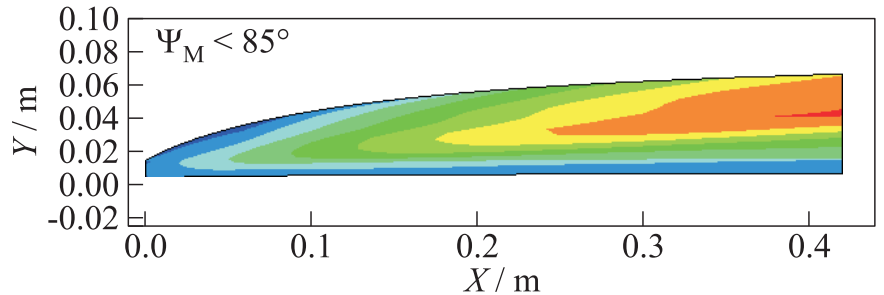

(b)

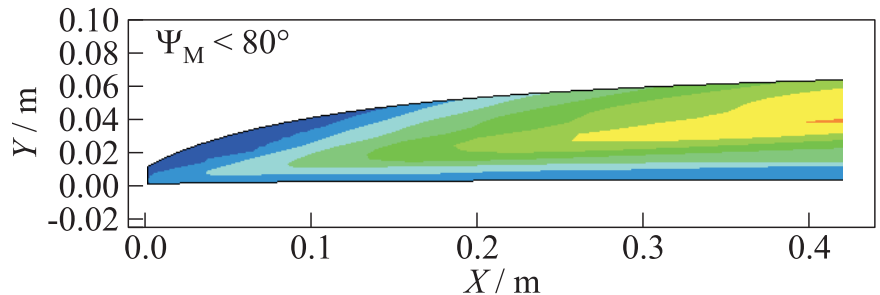

(c)

Figure $12 N$ factor of transition at $\mathrm{M}_{\infty}=6$. (Refer Orlik et al., p. 251.) 
close to the nose. Situation is less clear at $\mathrm{M}_{\infty}=6$ since the predicted transition depends on the amount of crossflow instability taken into account in the calculation of $N$ factors. Further comparison between calculated and experimental transition will help to get more specific information on this question.

\section{REFERENCES}

1. Saric, W. S., E. Reshotko, abd D. Arnal. 1998. Hypersonic laminar-turbulent transition. AGARD Report 319. 2:2-1-2-27.

2. Kornilov, V.I. 2009. Investigation of boundary-layer transition on flat plate at super- and hypersonic velocities. J. Thermophys. Aeromech. 16(3):365-73.

3. Lebiga, V.A. 2001. Boundary layer transition and reversal phenomenon at high flow velocities. 4th Pacific Conference (International) on Aerospace Science and Technology. Taiwan.

4. Jillie, D. W, and E. J. Hopkins. 1961. Effects of Mach number, leading-edge bluntness and sweep on boundary-layer transition on a flat plate. NASA Techn. Note D-1071:1-33.

5. Potter, J. L., and J.D. Whitfield. 1962. Effects of slight bluntness and roughness on boundary-layer transition in supersonic flows. J. Fluid. Mech. 12(4):501-35.

6. Owen, F. K. 1970. Transition experiments on a flat plate at subsonic and supersonic speeds. AIAA J. 8(3):518-23.

7. Ferrier, M. 2008. Analyse de la Stabilité et Prévision de la Transition Laminaire/Turbulent de l'Ecoulement Proche Paroi sur l'Avant-Corps d'un Véhicule Hypersonique. Thèse de l'Université d'Orléans.

8. Mack, L. M. 1975. Linear stability theory and the problem of supersonic boundarylayer transition. AIAA J. 13(3):278-89.

9. Ferrier, M., I. Fedioun, E. Orlik, and D. Davidenko. 2008. Transition prediction of the 3D boundary layer under an hypersonic vehicle forebody. 15th AIAA Space Planes and Hypersonic Systems and Technologies Conference (International). Dayton, Ohio.

10. Ferrier, M., I. Fedioun, E. Orlik, and D. Davidenko. 2009. Modal linear stability of the near-wall flow on a hypersonic forebody. J. Spacecraft Rockets 46(1):51-66. doi: $10.2514 / 1.37093$.

11. Kimmel, R.L., M. A. Klein, and S. N. Schwoerke. 1997. Three-dimensional hypersonic laminar boundary-layer computations for transition experiment designs. J. Spacecraft Rockets 34(4):409-15.

12. Arnal, D. 1998. Transition prediction in industrial applications. In: Transition, turbulence and combustion modeling. Eds. A. Hanifi, P. H. Alfredsson, A. V. Johansson, and D. S. Henningson. ERCOFTAC ser. Kluwer Academic Publs.

13. Ferrier, M., E. Orlik, I. Fedioun, and D. Davidenko. 2007. Three dimensional linear stability analysis of the boundary and entropy layers on hypersonic vehicle forebody. 2nd European Conference for Aerospace Sciences. Brussels, Belgium. 